\title{
DOPANT REDISTRIBUTION AND ACTIVATION IN THIN FILM SOI/SIMOX SUBSTRATES
}

\author{
A. K. Robinson, U. Bussmann, P. L. F. Hemment \\ Department of Electronic and Electrical Engineering, University of Surrey, \\ Guildford, Surrey, GU2 5XH, UK. \\ V. Sharma, J. A. Kilner \\ Department of Materials, Imperial College, Prince Consort Road, London, SW7 2BP, UK.
}

The high quality of SOI/SIMOX substrates and the inherent good control and reproducibility of the process have established SIMOX as the front running SOI technology. The thin silicon films have good crystal perfection and generally form a planar, abrupt interface with the underlying dielectric. As a result high performance circuits, showing improved speed and radiation tolerance over bulk silicon structures, have been successfully fabricated in these substrates and the expectation is that following processing and device optimization further circuit improvements will be achieved. In the future it may be possible to use this material for other novel and unconventional device structures. However optimization calls for detailed structural and electrical characterization of the material, including evaluation of the transport properties of the thin silicon films.

In this paper we report experiments to determine the transport properties, electrical activity and redistribution of dopants implanted into SIMOX samples with different silicon layer thicknesses.

High temperature annealed SIMOX samples with silicon film thicknesses of $2000 \AA$ (SIMOX1) and $3000 \AA$ (SIMOX2) have been implanted with $\mathrm{As}^{+}, \mathrm{Sb}^{+}, \mathrm{B}^{+}$and $\mathrm{P}^{+}$ions. The ion energies were chosen so that the projected range was similar for each species, being $\approx 500 \AA$. Doses were chosen to be similar to source/drain implantations used in CMOS devices. Activation of the dopant was achieved by annealing samples at either $950^{\circ} \mathrm{C}$ or $1150^{\circ} \mathrm{C}$ in flowing nitrogen gas in a resistivity heated furnace. The lower temperature was chosen to fully activate the dopant whilst the higher temperature allowed redistribution and segregation of the dopant to be studied. In addition, samples were isochronally ( $15 \mathrm{mins}$ ) annealed over the temperature range $200^{\circ} \mathrm{C}$ to $1150^{\circ} \mathrm{C}$. Surface analysis techniques included $1.5 \mathrm{MeV} \mathrm{He} \mathrm{He}^{+}$Rutherford backscattering (RBS) and channelling, SIMS and cross-sectional TEM whilst electrical data was obtained by Four Point Probe and Van der Pauw techniques. Dopant profiles have been compared with SUPREM simulations.

Figure 1 typifies the results and shows As concentration depth profiles obtained from samples prepared from bulk silicon, SIMOX1 and SIMOX2, which have been implanted with a dose of $5^{*} 10^{15} \mathrm{As}^{+} \mathrm{cm}^{-2}$ at $70 \mathrm{keV}$ and annealed at $950^{\circ} \mathrm{C}$ for 30 mins (curve (ii)) or $1150^{\circ} \mathrm{C}$ for 30 mins (curve (iii)). The profiles from the samples annealed at $950^{\circ} \mathrm{C}$ are the same, within experimental errors, confirming that similar diffusional broadening of the implanted profile (curve (i)) has occurred. Sheet resistance measurements show similar levels of dopant activation. In contrast, during the high temperature anneal, significant indiffusion of As occurs for bulk silicon whilst the SIMOX samples have essentially flat dopant profiles whose width is defined by the thickness of the silicon overlayer (marked by arrows in Figure 1(b),(c)). The uniform doping level and the absence of a concentration build up due to segregation at the $\mathrm{Si} / \mathrm{SiO}_{2}$ interface confirms 
the high quality of the material.Values of the Hall mobility are 49 and $57 \mathrm{~cm}^{2} \mathrm{v}^{-1} \mathrm{sec}^{-2}$ in SIMOX1 and SIMOX2, respectively, the differences being accounted for by the dependence upon carrier concentration.

Dopant activation during isochronal annealing is exemplified in Figure 2 which shows the temperature dependence of the sheet resistance following $\mathrm{As}^{+}$ion implantation into the same set of samples. The main difference is seen above $800^{\circ} \mathrm{C}$ when significant As diffusion occurs, which leads to uniform doping in the silicon layer and a value of sheet resistance which is temperature independent above $1000^{\circ} \mathrm{C}$.

Depth profiles determined by RBS and SIMS will be presented for the other dopants B, $P$ and Sb. The behaviour of all four dopants in SIMOX material will be evaluated and the consequent impact upon device geometries will be discussed.

\section{References}

[1] A. J. Auberton-Herve, Symposium on SOI Technology and Devices, ECS Meeting, Montreal, May 1990.

[2] J. Margail, J. Stoemenos, C. Jaussaud and M. Bruel. Appl. Phys. Lett. 54 (6) 1989.

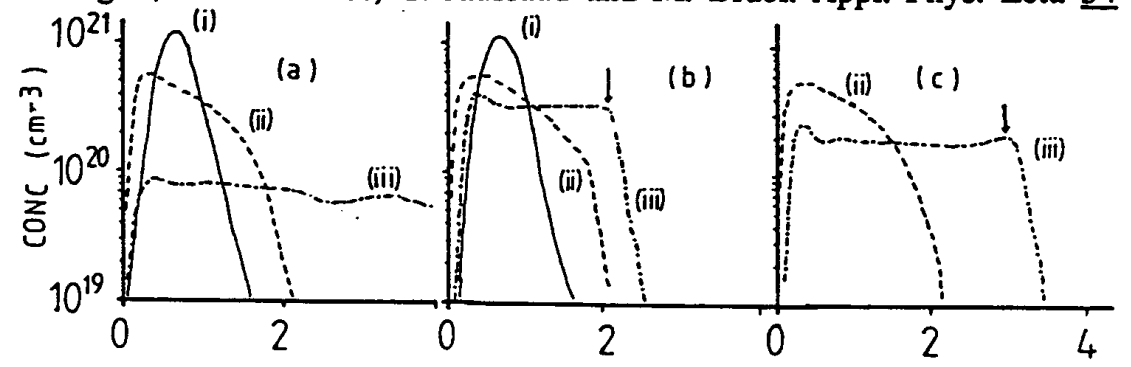

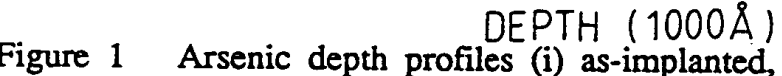

Figure 1 Arsenic depth profiles (i) as-implanted, (ii) $950^{\circ} \mathrm{C} 30$ mins and (iii) $1150^{\circ} \mathrm{C}$ $2 \mathrm{hrs}$, in (a) bulk silicon, (b) SIMOX with $2000 \AA$ of silicon overlay and (c) SIMOX with $3000 \mathrm{~A}$ of silicon overlay. Samples implanted with $5^{*} 10^{15} \mathrm{As} \mathrm{cm}^{-2}$ at $70 \mathrm{keV}$.

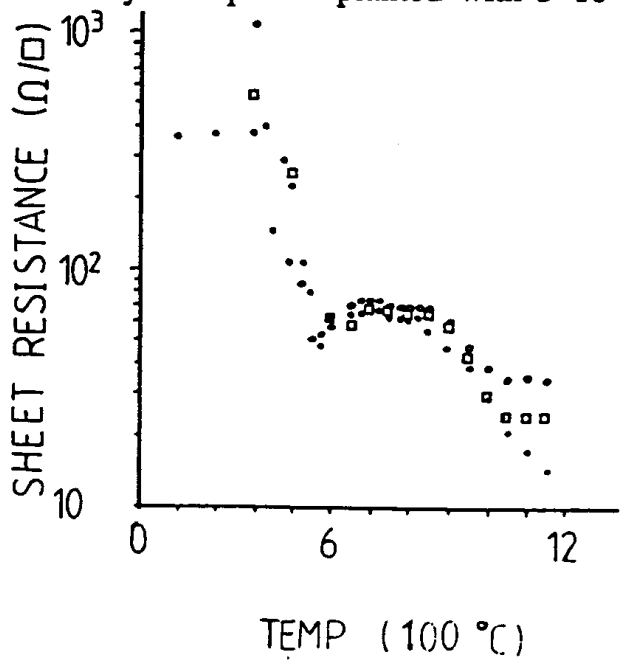

Figure 2 Dependence of sheet resistance upon isochronal anneal temperature for ( $\bullet$ bulk silicon, ( 0 ) SIMOX with $2000 \AA$ and ( $a$ ) SIMOX with $3000 \AA$ of silicon overlay.All samples implanted with $5^{*} 10^{15} \mathrm{As} \mathrm{cm}^{-2}$ at $70 \mathrm{keV}$. 